\title{
Primary Care Groups and Trusts: a threat or an opportunity for the development of community-based nursing in England
}

\author{
THERESE DOWSWELL PhD,${ }^{1}$ DAVID WILKIN PhD,${ }^{2}$ SUE KIRK m sc ${ }^{3}$ and JENNIFER BANKS-SMITH в A (Hons $)^{4}$ \\ ${ }^{1}$ Senior Research Fellow, ${ }^{2}$ Professor of Health Services Research, ${ }^{3}$ Research Fellow, National Primary Care Research \\ and Development Centre, University of Manchester, Manchester, UK, and ${ }^{4}$ Project Officer, Primary Care \\ Programme, King's Fund, London, UK
}

Correspondence

Therese Dowswell

National Primary Care Research

and Development Centre

5th Floor Williamson Building

University of Manchester

Oxford Road

Manchester M13 9PL

UK
DOWSWELL T., Wilkin D., KIRK S. \& BANKS-SMith J. (2002) Journal of Nursing Management 10, 283-289

Primary Care Groups and Trusts: a threat or an opportunity for the development of community-based nursing in England

Aims of the study: To examine the role of Primary Care Groups and Trusts (PCG/T) in relation to nurses working in general practice and community health services.

Background: Over the past two decades there have been rapid changes in the numbers and roles of nurses working in primary care and community based settings. The establishment of Primary Care Groups offers health care professionals, including nurses, the chance to develop local primary care services and to integrate community and primary care nursing. These developments may offer opportunities or pose threats to nursing staff.

Research methods: Data are drawn from a longitudinal study of a randomly selected sample of Primary Care Groups in England $(n=72)$. In a second survey of Groups carried out in autumn/winter 2000, Primary Care Group chairs and chief officers were interviewed by telephone.

Results: Response rates were $97 \%$ for both chairs and chief officers (69 of each). Chairs indicated that in most areas Primary Care Groups were consulting with local nurses to develop policy. Fifty-seven $(85 \%)$ reported that investment in nursing staff and nursing services was a high priority in their area. Twenty-eight $(41 \%)$ indicated that nurse-led services designed to increase patient access had already been established in their area, and 20 (29\%) were planning new nurse-led services. Many developments had been initiated by Primary Care Groups. Initiatives to integrate community and general practice based staff were underway in most areas.

Conclusions: Primary Care Groups and Trusts are initiating changes in general practice and community based services which are likely to have long-term and important implications for nurses in terms of their roles, conditions of work and future careers. It is important that nurses are consulted and are involved in developing and implementing policy change.

Keywords: community nursing, general practice, health policy, primary care

Accepted for publication: 18 October 2001 


\section{Introduction}

Community and primary care nursing have developed quite independently in the UK and the two groups of nursing staff continue to be managed and work separately (Damant et al. 1994, Hennessy 1997, McDonald et al. 1997). Since the establishment of the National Health Service (NHS) general practitioner (GP), hospital and community services have been organized and managed separately and until relatively recently very few nurses have been employed within general practices. Since the mid-1960s increasing numbers of nurses have been employed and managed by GPs although $70 \%$ of their salary costs are reimbursed by the NHS (Koperski et al. 1997). In contrast, community nurses have been employed directly by the NHS, and more recently by NHS Trusts with specific responsibilities for community health services. This difference in employers has implications for their management, roles, employment conditions and continuing professional development (Jenkins-Clarke et al. 1998, Williams \& Sibbald 1999).

Over the past two decades there have been particularly rapid changes in the numbers and roles of nurses working in general practice and community based settings (Broadbent 1998). MacDonald et al. (1991) have pointed to recent changes in the role of community nurses occurring as a result of earlier transfer of patients from hospital and of broader changes in general practitioner services. At the same time, there has been a major expansion in the number of nurses working in general practice and their role now includes health promotion and chronic disease management (Eve et al. 2000). New specialist roles have developed for nurses working in both general practice and in the community (Jones \& Mullee 1995, McDonald et al. 1997, JenkinsClarke et al. 1998, Reveley 1998).

The 1997 White Paper The New NHS: Modern. Dependable proposed major changes in the organization of primary and community health care services (Department of Health 1997), including the establishment of Primary Care Groups (PCGs). These groups serve localities of approximately 100000 people and have responsibility for developing primary and community health care services and improving the health of local people. PCGs were established with the intention that health care professionals (including nurses) would be in the 'driving seat' in planning and implementing service developments. While primary care group boards are dominated by GPs (with seven GP and two nurse members) board membership offers nurses a voice in policy formulation at the local level. Initially 481 PCGs were set up as sub committees of local health authorities. Over time it was envisaged that their level of responsibility would increase until they achieved Primary Care Trust (PCT) status with responsibility for directly providing primary and community health services and for commissioning hospital care.

The introduction of Primary Care Groups and Trusts (PCG/T) signalled a shift away from the market approach which had characterized the health care reforms of previous conservative governments (Bartlett et al. 1998). The reforms emphasized the need to reduce inequalities in health and inequities in service provision, to break down interprofessional boundaries, to foster multiprofessional and interagency communication and to co-ordinate care delivered by different professional groups. The separation of general practice and community services was perceived as a factor leading to service duplication, fragmentation and inefficiency and to inconsistent and discontinuous care for patients and the White Paper called for their integration.

Primary Care Groups have been charged with the task of implementing policies to 'modernize' primary and community care. These include promoting co-operation and partnership between general practices in order to reduce variation in services, improving access and extending the range of services available in primary care. Groups also face the task of integrating community and primary care to provide more seamless care for patients. These policy objectives have been reiterated in the more recent NHS Plan (Secretary of State for Health 2000).

This modernization agenda has direct and far-reaching implications for nurses (as clinicians and managers) working in both primary and community care. First, initiatives to improve access and increase the range of services available in primary care are likely to involve nursing staff. Innovations in this area include telephone advice and triage, personal medical services (PMS) pilot schemes, walk-in centres and minor injuries clinics. Where new services have already been established they have often been delivered by nurses (Jones et al. 1998, Wilkie \& Logan 1999, Chapple et al. 2000, Jones 2000, Lattimer et al. 2000). However, many such schemes were set up in response to GP recruitment problems and increasing patient demand, rather than as a planned development of nursing services and roles. Such initiatives are likely to increase with the advent of PCG/Ts. Secondly, the drive to increase collaboration between general practices is also likely to involve nurses. Nurses with specialist skills are a valuable resource within primary care and could potentially work across practices. Finally, the integration of community and primary care services is likely to have an impact on the future of 
both practice and community nurses. In some areas models of partnership and integration are already being developed but there is no template for successful integration and there are likely to be a range of local approaches (David \& Hopkins 1998, Galvin et al.1999). Potentially, as part of integration, community and practice nurses will be employed by the same employer and will have common management structures. Arguably, joint, multiprofessional community and general practice records are fundamental to successful integration (Clark \& Mooney 1999). All of these developments are likely to have an important impact on nursing roles, training and conditions of service. Nurses, as part of the wider constituency of staff working in community and primary care settings, thus have a key role in developing and implementing the modernization agenda set out in the White Paper (Department of Health 1997).

Primary Care Groups have now been established for 2 years and many are seeking, or have already achieved, Trust (PCT) status. It is now timely to consider how they are going about delivering these policy changes.

In this paper the focus will be on the role of nurses in service modernization and issues arising from the proposed integration of primary care and community services. The following questions will be addressed:

- Have nurses working in community and primary health care been consulted by PCGs with respect to changes that are likely to affect them?

- How much impact has consultation with nurses had on the development of PCG/T policy?

- How are PCG/Ts going about increasing collaboration, improving access and extending the range of services in primary care, and do initiatives involve nurses?

- How are PCG/Ts tackling the integration of community and primary care nursing?

\section{Methods}

\section{The National Tracker Study}

The data presented in this paper are drawn from the second of three, national surveys of a $15 \%$ (72) sample of the 481 PCGs established in 1999. The work has been funded by the Department of Health and is still in progress (the third survey will take place in Spring 2002). The broad aims of the study are to describe the way that PCG/Ts are tackling their core functions and to evaluate their progress.

Methods have been described in detail elsewhere (Wilkin et al. 2000) In brief, in this longitudinal survey of PCG/Ts survey methods were selected as they allow data to be collected in a standardized form from relatively large numbers of respondents. Data collection methods have included face-to-face and telephone interviews with PCG/T board members and postal questionnaires supplemented by local health care statistics and PCG/T reports and other documentation. The 72 PCG sites included were a stratified random sample of all PCGs in England and are broadly representative of all PCGs in terms of a number of criteria (e.g. local levels of deprivation and the size of the populations served by PCGs). The sample size was the largest achievable within resource constraints (Wilkin et al. 2000). The board members surveyed and the methods of data collection used have varied at different data collection points to take account of emerging policy issues and resource constraints (e.g. telephone interviews replaced face-to-face interviews in the second survey).

The first survey carried out in the winter of 1999 included interviews with PCG chairs and chief officers and postal questionnaires to board members, including nurse representatives. Results from the first tracker survey have been described elsewhere (Wilkin et al. 2000). One of the key findings from this survey was that PCG chief officers and chairs were perceived as being the most influential board members in terms of PCG decision-making. A second important finding was that the development of primary care services and achieving PCT status were identified as the key tasks for the year ahead (Wilkin et al. 2000). These issues are likely to have important implications for nurses working in primary care settings. Many new services in primary care are likely to be delivered by nurses and the achievement of PCT status in many cases implies the integration of community and primary care nursing.

In this paper, results are derived from the second survey which took place in the late autumn of 2000. At this time, for reasons of economy, only selected board members were surveyed and the sample did not include individual GP and nurse board members. As part of the second survey, telephone interviews were carried out with PCG/T chairs (predominantly GPs) and chief officers. Arguably, these are the most influential board members in terms of policy development, including those developments which are likely to have an impact on nursing staff. As part of these interviews respondents were specifically asked about consultation with nurses working in primary care settings, the development of primary care services involving nursing staff and the key issue of the integration of community and practice based nursing staff. 


\section{Results}

Full details of the response rates to the second tracker survey are set out elsewhere (Wilkin et al. 2001). By the autumn of 2000, two of the original 72 PCG/Ts included in the sample had merged. Response rates to the telephone interviews were $97 \%$ for both the PCG/T chairs (69/71) and chief officers (69/71). Complete data were available for most questions. Results will be presented under headings relating to the questions set out in the introduction.

\section{Consultation with and impact of nurses on PCG/T policy development}

Interviews with chairs covered the topic of consultation with nurses on key policy issues including service development and commissioning, prescribing, clinical governance and workforce integration.

Almost two-thirds of chairs $(42,64 \%)$ thought that nurses' views were well represented in PCG/T discussions, $22(33 \%)$ that nurses' views were represented moderately well and only $2(3 \%)$ that nurses' views were not well represented. Most PCG/Ts claimed to have consulted local nurses about key areas of policy and service development (Figure 1).

Whether or not PCG/T consultation with nurses actually affects decision making in PCG/Ts is another issue. Over half of the PCG/T chairs $(35,54 \%)$ thought

\section{Percentage of areas where nurses had been consulted on specific policy issues}

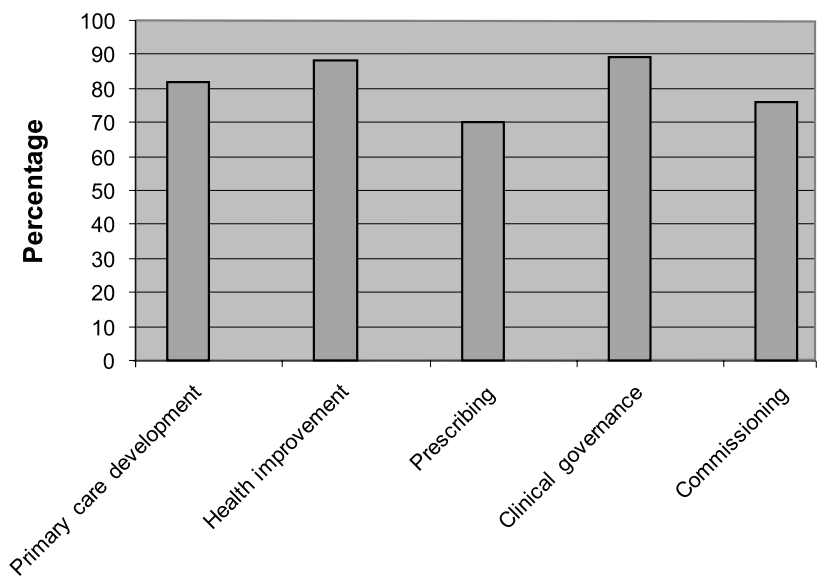

\section{Policy issues}

\section{Figure 1}

Percentage of $\mathrm{PCG} / \mathrm{T}$ chairs saying that community and primary care nurses had been consulted regarding specific policy issues: commissioning services, 50 (76\%); primary care development, 54 (82\%); health improvement, 58 (88\%); prescribing, 46 (70\%); clinical governance, 59 (89\%). that attempts to consult with nurses had been effective, $26(39 \%)$ that they had been moderately effective and only $4(6 \%)$ thought that attempts to consult had been ineffective.

Where nurses had been consulted, chairs assessed the impact of consultation on decision-making (Table 1).

Table 1 indicates that where nurses had been consulted (overall, in approximately $80 \%$ of PCG/Ts) the impact on decisions was variable. With regard to prescribing, for example, the impact of nurses was generally regarded as being limited or moderate, whereas nurses appeared to be having an important influence on decisions around clinical governance.

\section{Developments to improve services, increase collaboration and improve access}

Primary Care Groups and Trusts have an important role in primary care service development through increasing collaboration between local GP practices, promoting service developments to improve access and increasing the range of services available in primary care. In 57 areas $(85 \%)$ investment in nursing was rated as a high or very high priority for the PCG/T. Nurse-led services were already up and running in $41 \%$ (28) of areas and were planned in $29 \%$ (20). Of the 28 established schemes, in 14 cases developments were a result of PCG/T activity. In 18 areas (26\%) specialist nurse posts had been established and new specialist posts were planned in $33(48 \%)$. In more than half of the areas $(38,55 \%)$ where posts had been established or planned, developments had been initiated by the PCG. There were also plans to employ nurse practitioners in many areas: such posts already existed in 19 areas $(28 \%)$ and new posts were planned in almost half of the PCG areas $(33,48 \%)$. Primary Care Groups played a part in initiating developments to employ nurse practitioners in $38(55 \%)$ areas.

In addition to services that were explicitly nurse-led, chairs indicated that a range of other new services was underway or planned, many of which were likely to be delivered by nurses. These included walk-in treatment centres, telephone advice lines and improved facilities for the treatment of minor injuries. Many new developments had occurred as a direct result of PCG/T activity. For example, walk-in treatment centres had already been set up in six PCG/Ts and a further 12 areas had definite plans to introduce new centres. Of these 18 existing or planned developments, eight had been initiated by the PCG/T. Thirty-three $(62 \%)$ of the personal medical services pilot schemes reported included nurseled services. 
Table 1

Impact of consultation with nurses on $\mathrm{PCG} / \mathrm{T}$ decisions

\begin{tabular}{lccc}
\hline & $\begin{array}{c}\text { Limited impact } \\
\text { (rated 1 or 2) }\end{array}$ & $\begin{array}{c}\text { Moderate impact } \\
\text { (rated 3) }\end{array}$ & $\begin{array}{r}\text { Important impact } \\
\text { (rated 4 or 5) }\end{array}$ \\
\hline Commissioning & $21(43 \%)$ & $15(31 \%)$ & $13(26 \%)$ \\
Primary care development & $13(24 \%)$ & $23(43 \%)$ & $18(33 \%)$ \\
Health improvement & $12(21 \%)$ & $28(48 \%)$ & $18(31 \%)$ \\
Prescribing & $21(47 \%)$ & $16(35 \%)$ & $8(18 \%)$ \\
Clinical governance & $13(22 \%)$ & $18(31 \%)$ & $28(47 \%)$ \\
\hline
\end{tabular}

Percentage figures relate to the numbers of PCG/Ts that did consult on this issue.
Many PCG/Ts had promoted schemes to increase collaboration between local GP practices. Nurses with specialist skills were increasingly working across practices. In $43 \%$ of areas (30) specialist nurses were already working in more than one practice and in $26 \%$ (18) there were definite plans to increase this type of collaboration. Of the existing collaborative arrangements or planned schemes, $26(38 \%)$ had been initiated by the PCG/T.

\section{Integration of the community and primary care nursing workforces}

Results from the interviews with PCG/T chief officers suggest that service integration is a key issue for PCGs as they move towards Trust status. Fifty-one of the chief officers $(74 \%)$ mentioned the integration of the nursing workforces as one of the three most important reasons for becoming a Trust. The majority $(58,84 \%)$ were planning to provide community nursing services so that the PCT would become the employer of community nursing staff.

The levels of support for seeking Trust status amongst local community and primary care nurses varied. It was generally perceived that most nurses supported the idea. No chief officers perceived that local nurses were totally opposed to the scheme, $23(35 \%)$ perceived that there was moderate support for seeking changed status and $40(63 \%)$ that local nurses positively supported moves towards becoming a Trust. Three respondents said they did not know whether or not a change in status was favoured by nursing staff.

In many areas, schemes were already underway or planned to integrate the work of practice and community nurses (Table 2 ).

\section{Table 2}

Initiatives to promote the integration of practice and community nurses $(n=69)$

\begin{tabular}{lccc}
\hline Scheme & $\begin{array}{c}\text { Scheme already } \\
\text { underway }\end{array}$ & Scheme planned & $\begin{array}{c}P C G / T \text { initiated } \\
\text { schemes }\end{array}$ \\
\hline Common contracts for staff & $5(7 \%)$ & $16(23 \%)$ & $10(14 \%)$ \\
Common management & $5(7 \%)$ & $18(26 \%)$ & $16(23 \%)$ \\
Integrated nursing teams & $21(30 \%)$ & $31(45 \%)$ & $34(49 \%)$ \\
Joint meetings & $57(83 \%)$ & $6(9 \%)$ & $38(55 \%)$ \\
Shared records & $21(30 \%)$ & $12(17 \%)$ & $15(22 \%)$ \\
Joint training & $40(58 \%)$ & $17(25 \%)$ & $41(59 \%)$ \\
\hline
\end{tabular}

In most of the areas, practice and community nurses were still being managed separately but almost a third of areas were moving towards common contacts and common management. In a minority of areas $(18,26 \%)$ there were no initiatives underway or planned to promote the integration of community and nursing staff.

\section{Conclusions}

In this paper we have drawn on interviews with chief officers and chairs from a sample of PCG/Ts. Overall, the sample was not large, although it represented $15 \%$ of all English PCG/Ts and response rates were high. However, as leading figures within the organizations it is arguable that chairs and chief officers would take a more positive view of the work of PCG/Ts and the level of support for it than others outside the board. There is also some evidence that within PCG/T boards different group members may not share the same priorities and these priorities, in turn, may differ from those of local health care staff outside PCG/T boards (Lucas \& Bickler 2000). It is possible that the views of PCG/T chairs and chief officers may have differed from those of nurse board members. This may well be true, however, evidence from the first Tracker survey suggests that the former are likely to be the most influential in terms of policy developments and this is likely to apply to policy relating to nursing issues as well as other areas. Thus, despite limitations, our study provides useful evidence of the early activities of these new organizations and the implications for nurses and nursing.

The fact that nurses are represented on boards and that efforts are being made to consult nurses working in the locality seems a positive step. Chairs of PCG/Ts felt their attempts to consult with nurses had been 
mainly effective, with consultation taking place on key issues in local health policy development. Nurses now have mechanisms to contribute to policy formulation in primary and community health care. As Fatchett (1998) has noted '... in their own interest nurses have to be engaged in the changes that are taking place, and which will shape their role and the way it interacts with others in society. Passive indifference or sullen opposition are not options...' (pp. 157-158). Nurse representation on PCG/T boards offers an opportunity to nurses. It is possible that the dominance of GPs within boards may constitute a threat. However, PCG/ $\mathrm{T}$ decision-making is not driven by personal and professional factors alone. Decision-making is taking place within the context of a local health services infrastructure and a national policy agenda. The influence of any individual board member is likely to be tempered by this broader framework.

The introduction of PCGs has increased opportunities for nurses to develop new roles and services. In many areas new nurse-led services had been planned or were already underway and many schemes had developed as a result of PCG/T activity. These initiatives to improve access and to extend the range of services may present many opportunities for nurses although their implications for individual nurses are likely to vary. Some nurses may be expected to take on new work without appropriate recognition (in terms of pay or promotion) or without being able to shed any of their existing workload and some may feel ill-prepared to take on new or extended roles. One implication arising from the development of new specialist posts may be an increasing differentiation in roles. These specialist roles potentially offer nurses opportunities to develop new skills, work in new areas and deliver improved services to patients but may lead to problems with communication and co-ordination and less holistic care.

The trend towards integrating community and practice nursing was strong. Community and practice nurses are meeting and training together, and in some areas common management structures and contacts have been introduced. These findings raise questions which we have not been able to address. Qualitative work is needed to examine in detail the views of community and practice nurses on integration. There seems to be general support for integration amongst nurses although it is not yet clear what difference it will make. Whether service integration represents a threat or an opportunity for nurses begs the question of what exactly is meant by integration. In the past, in situations where nurses have worked in integrated teams, integration may not have been initiated by nurses (Goodman 2000). At the same time, in situations where nurses have been managed separately there has, nevertheless, been some collaboration. Most community nurse already have access to their patients' general practice records, and many share premises with practice nurses and general practitioners. Closer working between community and practice nurses may mean improved care for patients with greater consistency and less duplication. It is not yet clear whether integration will increase medical control over nursing or, conversely whether it will strengthen nursing control over professional and service developments in primary care.

Primary Care Groups and Trusts are charged with the tasks of implementing the integration of community and primary care, improving health, developing services and commissioning care. Their establishment offers nurses a broad range of opportunities: to develop new skills, roles and responsibilities, to collaborate with other health care providers and to improve the range and quality of care available to patients. General practitioner dominance of PCG/Ts and the possibility of role fragmentation may pose threats to nurses and it is important that service developments are adequately planned and resourced so that nurse-led services are not regarded as a means of plugging the gap left by GP recruitment problems. It is vitally important for nurses to seize the opportunities available to them by taking a leading role in planning and implementing any changes that affect them or their patients.

\section{References}

Bartlett W., Roberts J.A. \& LeGrand J. (1998) A Revolution in Social Policy: Quasi-Market Reforms in the 1990s. Policy Press, Bristol.

Broadbent J. (1998) Practice nurses and the effects of the new general practitioner contract in the British NHS: the advent of a professional project? Social Science and Medicine 47, 497-506.

Chapple A., Rogers A., MacDonald W. \& Sergison M. (2000) Patients' perceptions of changing professional boundaries and the future of 'nurse-led' services. Primary Health Care Research and Development 1, 51-59.

Clark J. \& Mooney G. (1999) Primary health care records: an integrated approach. Nursing Times 95 (16), 48-50.

Damant M., Martin C. \& Openshaw S. (1994) Practice Nursing: Stability and Change. Mosby, Guilford.

David A. \& Hopkins S. (1998) Model partnerships. Nursing Times 94 (23), 38-40.

Department of Health (1997) The New NHS: Modern, Dependable. The Stationery Office, London.

Eve R., Hodgkin P., Quinney D. \& Waller J. (2000) What Do Practice Nurses Do? A Study of Roles, Responsibilities and Patterns of Work. The Centre for Innovation in Primary Care, Sheffield. 
Fatchett A. (1998) Nursing in the New NHS: Modern, Dependable? Bailliere, Tindall.

Galvin K., Andrewes C., Jackson D., Cheesman S., Fudge T., Ferris R. \& Graham I. (1999) Investigating and implementing change within the primary health care nursing team. Journal of Advanced Nursing 30, 238-247.

Goodman C. (2000) Integrated nursing teams: in whose interests? Primary Health Care Research and Development 1, 207-215.

Hennessy D. (1997) Community Health Care Development. Macmillan, London.

Jenkins-Clarke S., Carr-Hill R. \& Dixon P. (1998) Teams and seams: skill mix in primary care. Journal of Advanced Nursing 28, 1120-1126.

Jones M. (2000) Walk-in primary medical care centres: lessons from Canada. British Medical Journal 321, 928-931.

Jones K. \& Mullee M. (1995) Pro-active nurse run asthma care in primary practice reduces morbidity: scientific fact or medical assumption. British Journal of General Practice 45, 497-499.

Jones K., Gilbert P., Little J. \& Wilkinson K. (1998) Nurses triage for house call requests in a Tyneside general practice: patients' views and the effect on doctor workload. British Journal of General Practice 48, 1303-1306.

Koperski M., Rogers S. \& Drennan V. (1997) Nurse practitioners in general practice - an inevitable progression? British Journal of General Practice 47, 696-698.

Lattimer V., Sassi F., George S., Moore M., Turnbull J., Mullee M. \& Smith H. (2000) Cost analysis of nurse telephone consulatation in out of hours primary care: evidence from a randomised controlled trial. British Medical Journal 320, 1053-57.

Lucas K. \& Bickler G. (2000) Altogether now? Professional differences in the priorities of primary care groups. Journal of Public Health Medicine 22, 211-215.
MacDonald L., Addington Hall J., Hennessy D. \& Gould T. (1991) Effects of reduction of acute hospital services on district nursing services: implications for quality assurance. International Journal of Nursing Studies 28, 247-255.

McDonald A., Langford I.H. \& Boldero N. (1997) The future of community nursing in the United Kingdom: district nursing, health visiting and school nursing. Journal of Advanced Nursing 26, 257-265.

Reveley S. (1998) The role of the triage nurse practitioner in general medical practice: an analysis of the role. Journal of Advanced Nursing 28, 584-591.

Secretary of State for Health (2000) The NHS Plan: A Plan for Investment. A Plan for Reform. Cm 4818-I. The Stationery Office, London.

Wilkie P. \& Logan A. (1999) Walk-in centres: caution not cynicism. British Journal of General Practice 49, 1017.

Wilkin D., Gillam S. \& Leese B. (eds) (2000) The National Tracker Survey of Primary Care Groups and Trusts: Progress and Challenges 1999/2000. National Primary Care Research and Development Centre, University of Manchester, Manchester.

Wilkin D., Gillam S. \& Coleman A. (eds) (2001) The National Tracker Survey of Primary Care Groups and Trusts 2000/ 2001: Modernising the NHS? National Primary Care Research and Development Centre, University of Manchester, Manchester.

Williams A. \& Sibbald B. (1999) Changing roles and identities in primary health care: exploring a culture of uncertainty. Journal of Advanced Nursing 29, 737-745. 